\title{
Nanoparticles for Cardiovascular Imaging and Therapeutic Delivery, Part 1: Compositions and Features
}

\author{
John C. Stendahl ${ }^{1,2}$ and Albert J. Sinusas ${ }^{1-3}$ \\ ${ }^{1}$ Section of Cardiovascular Medicine, Department of Internal Medicine, Yale University School of Medicine, New Haven, \\ Connecticut; ${ }^{2}$ Yale Translational Research Imaging Center, Yale University School of Medicine, New Haven, Connecticut; and \\ ${ }^{3}$ Department of Diagnostic Radiology, Yale University School of Medicine, New Haven, Connecticut
}

\begin{abstract}
Imaging agents made from nanoparticles are functionally versatile and have unique properties that may translate to clinical utility in several key cardiovascular imaging niches. Nanoparticles exhibit size-based circulation, biodistribution, and elimination properties different from those of small molecules and microparticles. In addition, nanoparticles provide versatile platforms that can be engineered to create both multimodal and multifunctional imaging agents with tunable properties. With these features, nanoparticulate imaging agents can facilitate fusion of highsensitivity and high-resolution imaging modalities and selectively bind tissues for targeted molecular imaging and therapeutic delivery. Despite their intriguing attributes, nanoparticulate imaging agents have thus far achieved only limited clinical use. The reasons for this restricted advancement include an evolving scope of applications, the simplicity and effectiveness of existing smallmolecule agents, pharmacokinetic limitations, safety concerns, and a complex regulatory environment. This review describes general features of nanoparticulate imaging agents and therapeutics and discusses challenges associated with clinical translation. A second, related review to appear in a subsequent issue of JNM highlights nuclear-based nanoparticulate probes in preclinical cardiovascular imaging.
\end{abstract}

Key Words: nanoparticles; cardiovascular disease; nuclear imaging; molecular imaging; theranostics

J Nucl Med 2015; 56:1469-1475

DOI: 10.2967/jnumed.115.160994

Cardiovascular diseases remain the most prominent worldwide cause of mortality (1). New generations of imaging agents are being designed to address the cardiovascular disease epidemic by advancing understanding of pathophysiology, improving detection and risk stratification, and enhancing treatment through targeted and image-guided therapies. Although most clinical imaging agents are smallmolecule compounds, nanoparticulate agents have garnered significant interest because of their distinct physical and biologic

\footnotetext{
Received Jan. 26, 2015; revision accepted Jul. 23, 2015.

For correspondence or reprints contact: Albert J. Sinusas, Yale University School of Medicine, 333 Cedar St., P.O. Box 208017, New Haven, CT 06520.

E-mail: albert.sinusas@yale.edu

Published online Aug. 13, 2015.

COPYRIGHT (c) 2015 by the Society of Nuclear Medicine and Molecular Imaging, Inc.
}

properties and significant functional versatility. Nanoparticulate imaging agents typically demonstrate pharmacokinetic and biodistribution behavior different from that of small molecules and provide flexible platforms for integration of multiple functional entities, including targeting ligands, therapeutics, and/or multiple types of contrast materials. Importantly, nanoparticulate imaging agents are also capable of amplifying signals by delivering large volumes of contrast materials in concentrated packages. Despite these intriguing attributes, nanoparticulate imaging agents have thus far attained only limited clinical use and require additional development to overcome various functional limitations and safety concerns.

This review describes general features of nanoparticulate imaging agents and discusses challenges associated with clinical translation. A second, related review to be published in a subsequent $J N M$ issue highlights specific preclinical applications of nuclear-based nanoparticulate probes in cardiovascular imaging.

\section{NANOPARTICLE COMPOSITIONS AND SIZE- BASED PROPERTIES}

A nanometer is one billionth of a meter $\left(10^{-9}\right)$, or about one-half the width of a DNA double helix. Although definitions vary, the term nanoparticle generally refers to particles with diameters on the scale of several to several hundred nanometers. Particles with these dimensions are on the size scale of biologic macromolecules and exhibit size-based physical and biologic properties that have potential utility in certain cardiovascular imaging niches. Numerous nanoparticle compositions have been used in preclinical nuclear imaging of cardiac and vascular structures, including micelles (2), liposomes (3), polymeric particles (4-8), dendrimers (9-11), lipoprotein particles (12), gold particles (13), iron oxide particles (14-16), perfluorocarbon emulsions (17), carbon nanotubes (18), and upconversion nanophosphors (Fig. 1) (19).

Because of their size, nanoparticulate imaging agents typically exhibit pharmacokinetic behaviors different from those of small molecule $(<1 \mathrm{~nm})$ and microparticulate $(>1$ $\mu \mathrm{m})$ agents $(20,21)$. These size-based differences arise from interactions of particles with structural features of the vasculature. Small and intermediate-sized molecules and small 


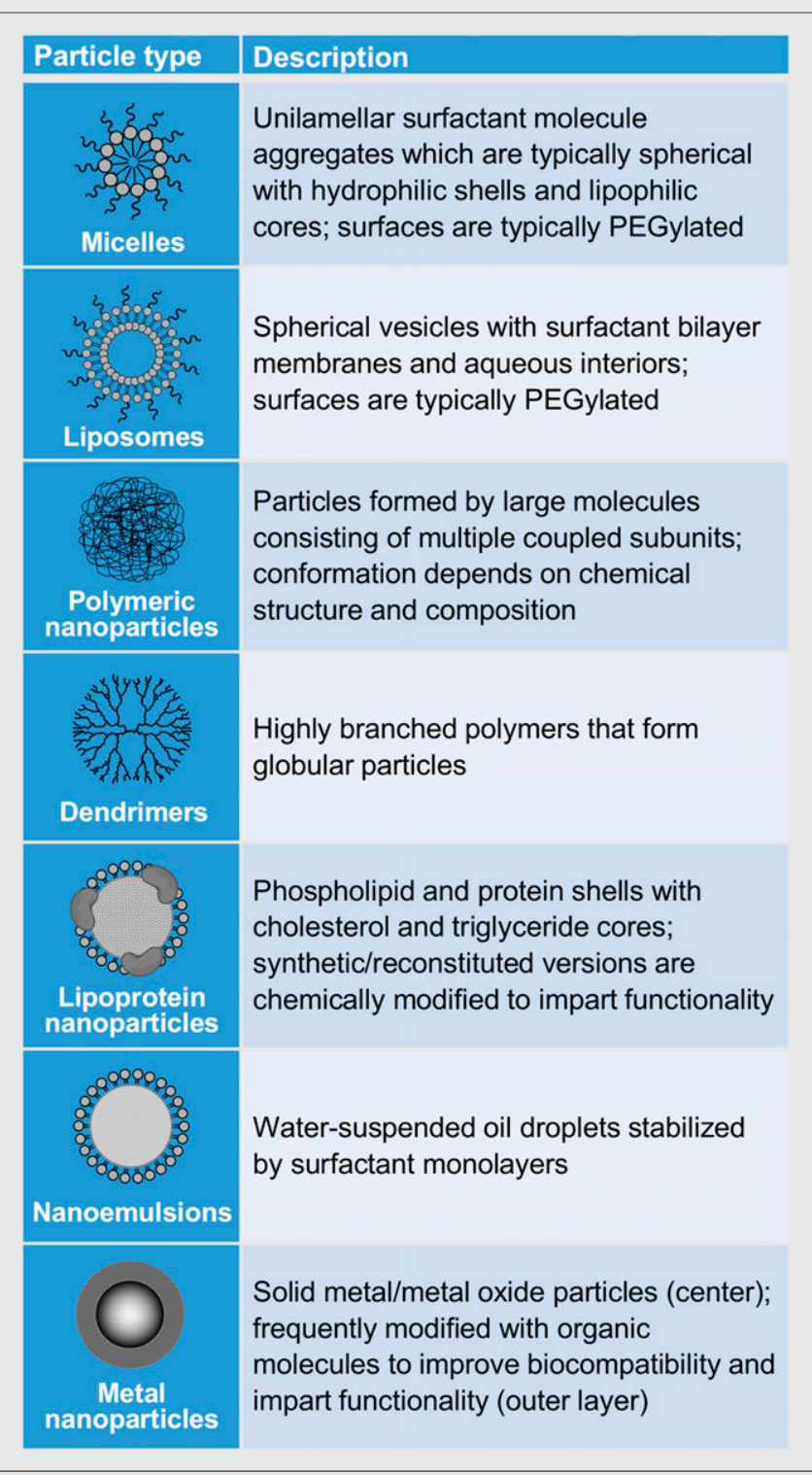

FIGURE 1. Select nanoparticle types used in preclinical cardiovascular imaging research and therapeutic delivery.

nanoparticles typically undergo relatively fast blood clearance by renal filtration, which has an approximate size limit of $5.5 \mathrm{~nm}$ (for clearance in $<4 \mathrm{~h}$ ) (22). Relatively fast blood clearance also occurs by filtration through fenestrae of hepatic sinusoids, which has an approximate size limit of $70 \mathrm{~nm}$ (23). In contrast, larger nanoparticles are more likely to be cleared from the blood by cells of the mononuclear phagocyte system (MPS) in organs such as the liver and spleen (23). In general, rates of MPS uptake increase with increasing particle size, although MPS uptake can be significantly reduced at intermediate particle sizes by surface modification with hydrophilic moieties such as ganglioside (23) or polyethylene glycol (24). For nanoparticles chemically modified in this fashion, sizes on the order of $100 \mathrm{~nm}$ have been shown to minimize the effects of the various blood clearance mechanisms and achieve the longest circulation times $(23,24)$.
The large size of nanoparticles in relation to smallmolecule agents also has important implications with regard to tissue uptake and distribution. Although small molecules typically extravasate through vascular endothelium at greater rates, they also tend to penetrate tissues more deeply and wash out more readily. In contrast, intermediately sized nanoparticles are generally too large for significant extravasation through healthy, nonfenestrated endothelium but often traverse more permeable vessels, such as sinusoidal capillaries of the liver and spleen, and those present in tumors and sites of inflammation and angiogenesis $(20,25)$. Because of their relatively large size, nanoparticles are less likely to undergo washout and thus tend to accumulate at greater rates in the perivascular spaces of these permeable tissues. The passive accumulation of nanoparticles in tissues with increased vascular permeability is known as the enhanced permeability and retention effect (EPR) and has been exploited in numerous therapeutic and imaging applications $(2,4,6,14,15)$. Although EPR can be useful for general detection of pathologic tissues, it is important to consider that the effect typically occurs over longer time scales (hours) that may not be conducive to clinical diagnostic imaging (25). Moreover, EPR-based nanoparticle accumulation often impairs particle elimination and is highly unfavorable for targeted molecular imaging applications because it produces nonspecific background signals that may obscure or confound desired signals from molecular binding events (26).

In addition to these size-based physiologic effects, nanoparticles exhibit certain size-based physical effects that also contribute to their utility as imaging and therapeutic agents. For example, quantum dots (27) and superparamagnetic particles (28) produce contrast by distinct mechanisms directly related to their size; these contrast-producing phenomena do not occur in small molecules or bulk materials of similar composition. Similarly, size-based physical effects also play important roles in nanoparticles designed for photothermal therapy (29).

\section{DESIGN VERSATILITY: MULTIMODAL AND MULTIFUNCTIONAL IMAGING AGENTS}

One of the most intriguing features of nanoparticles is their design flexibility. Nanoparticles provide versatile platforms for the creation of both multimodal and multifunctional imaging agents. The surfaces and cores of nanoparticles can be designed to incorporate multiple imaging materials as well as functional entities such as targeting ligands, therapeutics, and other agents that modify chemical and biologic properties.

Hybrid nanoparticles containing multiple imaging agents can potentially improve diagnostic utility by facilitating the integration of high-sensitivity and high-resolution imaging approaches $(11,17,30)$. Among current clinical imaging modalities, SPECT and PET provide the greatest sensitivity for detection and quantification of biochemical processes and scant molecular epitopes (Table 1) (31). However, these nuclear modalities provide poor spatial resolution and limited anatomic localization and are subject to significant partial-volume error. 
TABLE 1

Sensitivities and Spatial Resolutions of Standard Clinical Imaging Modalities (31)

\begin{tabular}{lcc}
\hline Modality & Sensitivity (mol/L) & Resolution (mm) \\
\hline CT & $10^{-2}-10^{-3}$ & $0.5-2.0$ \\
MR & $10^{-3}-10^{-5}$ & $0.5-1.5(1.5 \mathrm{~T}), 0.01-0.10(>3 \mathrm{~T})$ \\
SPECT & $10^{-10}-10^{-11}$ & $7-15$ \\
PET & $10^{-11}-10^{-12}$ & $6-10$ \\
\hline
\end{tabular}

To address this problem, PET and SPECT images are frequently acquired in combination with those of lower-sensitivity, highresolution techniques, such as CT and MR. The assimilation of agents for high-sensitivity and high-resolution imaging modalities in a single nanoparticle further expedites multimodal image acquisition and facilitates data integration. This capability is particularly useful for the detection and localization of sparse epitopes, such as those associated with angiogenesis and atherosclerosis. Lijowski et al. provided an early illustration of the diagnostic effectiveness of multimodal nanoparticulate imaging agents using $\alpha_{\nu} \beta_{3}$-targeted ${ }^{99 \mathrm{~m}} \mathrm{Tc}$-gadolinium perfluorocarbon nanoparticles to achieve highly sensitive detection and specific localization of tumor angiogenesis in rabbits via SPECT/CT/MR (17).

Nanoparticles can also be designed to encapsulate large payloads of contrast material and deliver them in targeted, concentrated packages. Although concentrated delivery of imaging agents is generally not necessary for targeted molecular imaging with highly sensitive nuclear modalities, it is often required for molecular imaging with less sensitive modalities such as CT and MR. In these modalities, nanoparticulate agents can potentially achieve significantly

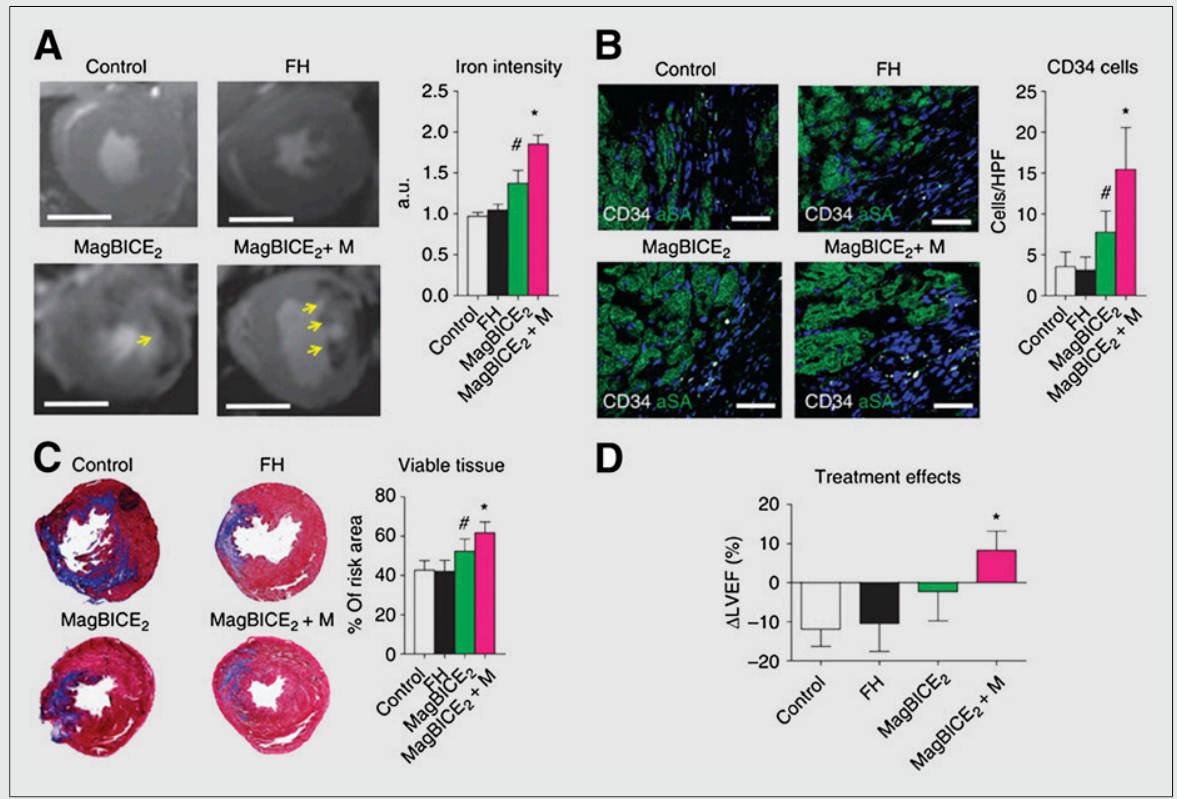

FIGURE 2. Treatment effects of theranostic magnetic bifunctional cell engager (MagBICE) nanoparticles administered to rats after myocardial infarction. MagBICE 2 nanoparticles are composed of dextran-coated iron oxide cores coupled to anti-myosin light-chain kinase and anti-CD34, which bind injured cardiomyocytes and CD34-positive peripheral blood mononuclear cells, respectively. FH denotes dextran-coated iron oxide nanoparticles without antibody conjugation. (A) $\mathrm{T}_{2}{ }^{*}$-weighted $\mathrm{MR}$ images and corresponding graphs showing specific targeting of $\mathrm{MagBICE}_{2}$ nanoparticles to infarcted myocardium and enhancement of targeting with localized magnetic field application ( $\left.\mathrm{MagBICE}_{2}+\mathrm{M}\right)$ $(n=3$ per group, scale bars represent $0.5 \mathrm{~cm}$ ). (B) Confocal micrographs and corresponding quantification of $\mathrm{MagBICE}_{2}$ and $\mathrm{MagBICE}_{2}+\mathrm{M}$-based recruitment of CD34-positive cells (white) to infarcts ( $n=3$ per group). (C) Masson trichome images and corresponding quantification of scarred (blue) and viable (red) myocardium 4 wk after myocardial infarction ( $n=3$ per group). (D) Change in left ventricular ejection fraction ( $\triangle$ LVEF) assessed by echocardiography 4 wk after myocardial infarction $(n=6$ per group). ( $P<0.05$ compared with control or FH group. ${ }^{*} P<0.05$ compared with any other groups.) (Reprinted with permission of (36).) greater signal amplification than small molecule agents by delivering greater quantities of imaging materials per targeted molecular binding event. In the case of nanoparticulate agents for MR, signals are further enhanced by optimizing particle structural features that influence molecular relaxation (32,33). Molecularly targeted nanoparticles have been used for preclinical MR imaging of structures such as angiogenic vessels (34), atherosclerotic plaques (35), and injured myocardium (Fig. 2) (36). Similarly, targeted nanoparticles have been used for CT imaging of structures such atherosclerotic plaques (37), thrombi (38), and myocardial scars (39), although the sensitivity of nanoparticleenhanced CT imaging remains on the low side for many molecular imaging applications.

Despite its relatively low sensitivity, CT remains a promising candidate for certain molecular imaging applications because of its faster scan times, suitability for quantitative analysis, and the emerging capabilities of multienergy and spectral CT imaging. Unlike traditional CT, spectral CT provides compositional data for imaged materials by analyzing their characteristic, energy-dependent x-ray absorption behavior (40). This additional information facilitates specific 


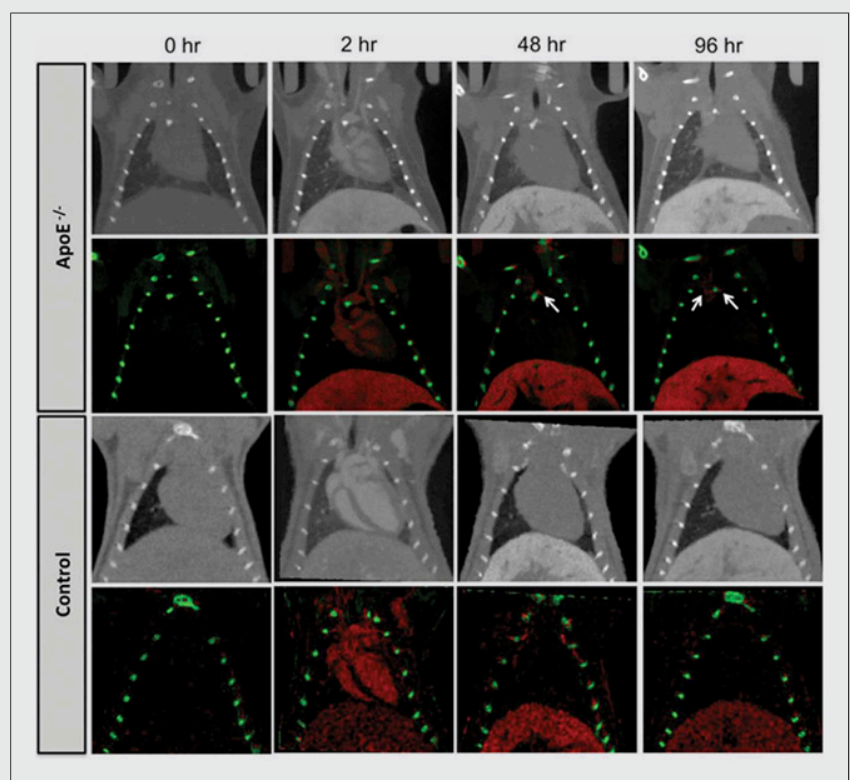

FIGURE 3. Complementary gray scale and spectrally decomposed dual-energy CT images of control and apolipoprotein E-deficient $\left(\mathrm{ApoE}^{-/-}\right)$mice before and after administration of a liposomal iodine contrast agent (red denotes iodine, green denotes calcium). Spectral decomposition permits more sensitive discrimination between plaque calcification and iodinated contrast. Arrows demonstrate colocalization of aortic plaque calcium and liposomal iodine taken up by plaque macrophages after blood pool clearance of liposomal iodine. (Reprinted with permission of (43).)

differentiation of tissue components and permits concurrent localization of multiple contrast-labeled molecular epitopes. Multienergy and spectral CT imaging has been used in several early studies to provide data on the composition of atherosclerotic plaques (Fig. 3) (41-43). Clinical translation of nanoparticle-enhanced spectral CT imaging requires continued development of spectral scanner technology, as well as new types of contrast media that contain adequate quantities of materials with K-shell absorption energies that lie within the clinical CT energy bandwidth (44). It is critical for patient safety that these high-atomic-number (high Z) materials are delivered in nonbioactive forms that can be subsequently bioeliminated in timely manners.

The ability of nanoparticulate imaging agents to incorporate and deliver therapeutic payloads is one of their most intriguing qualities. Nanoparticles with this feature are classified as theranostics because of their joint therapeutic and diagnostic functionality (45). The incorporation of both entities in a single particle enables capabilities such as imageguided interventions and direct monitoring of therapeutic agent distribution, metabolism, and biologic effects. Targeted theranostic nanoparticles have been developed for multiple potential clinical cardiovascular applications, including stabilization of atheromas via delivery of antiangiogenic (46) and antiinflammatory drugs (Fig. 4) (47,48), treatment of peripheral artery disease via delivery of angiogenic factors (49), and promotion of postinfarct cardiac repair by delivery of angiogenic genes (50) and antiinflammatory stimuli (51). In addition, nanoparticles have been used to track therapeutic cells delivered to infarcted myocardium (52). Aside from these applications, numerous therapeutic nanoparticles have been developed for the treatment of cardiovascular diseases and could conceivably be modified to incorporate clinically useful imaging media (53-56).

Importantly, nanoparticles can also be designed for targeted delivery to potentially enhance both diagnostic specificity and therapeutic efficacy. Passive nanoparticle targeting can be accomplished in certain tissues through the EPR effect and other size-based effects described above and is facilitated by surface chemistry modifications, such as the incorporation of polyethylene glycol to limit opsonization and nonspecific particle uptake $(2,8,11,14)$. In contrast, nanoparticles designed for active targeting are equipped with biologically active ligands such as peptides and antibodies that bind specific molecular elements of native tissue and transplanted cells $(5,9,10,12)$. Despite the attractiveness of this concept, achieving true molecular targeting with nanoparticles is often challenging because of competing EPR effects. Several studies have shown that, because of EPR effects, the incorporation of targeting ligands on nanoparticle surfaces often does not increase particle localization in targeted tissues, even in the presence of increased specific particle binding and internalization $(57,58)$. Moreover, the increased rate of nanoparticle internalization due to molecular binding does not always increase therapeutic efficacy, as drugs delivered by this method often remain trapped in nanoparticles or are degraded in lysosomes (59). Interestingly, true receptor-specific imaging in highly permeable tumor tissues has been demonstrated with small, renally cleared nanoparticles, which are apparently less susceptible

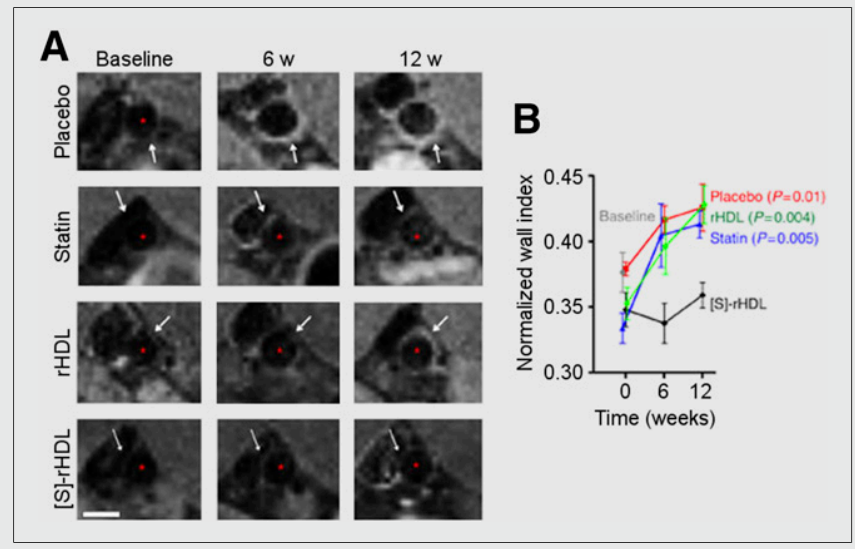

FIGURE 4. (A) Representative T1-weighted MR images of abdominal aortas in apolipoprotein E-deficient mice fed highcholesterol diets and treated with one of the following: intravenous saline (placebo), oral simvastatin (statin), reconstituted HDL nanoparticles (rHDL), or simvastatin-HDL nanoparticles ([S]-rHDL). Progressive aortic thickening was seen in all groups except those treated with [S]-rHDL (scale bar represents $1 \mathrm{~mm}$ ). (B) Normalized wall indices ([outer wall area - lumen area]/outer wall area) from MR images, demonstrating the substantial therapeutic effect of targeted simvastatin delivery via [S]-rHDL ( $n=8$ per group). (Reprinted with permission of (48).) 
to EPR effects because of their small size and more rapid clearance (26).

The potential clinical utility of nanoparticles in targeted molecular imaging and therapeutic delivery is illustrated in a recent study by Cheng et al. in which MR-imageable superparamagnetic iron nanoparticles were conjugated to two different types of antibodies: one to facilitate in vivo binding of particles to injured myocardium and another for subsequent capture and localization of endogenous or exogenous therapeutic cells (Fig. 2) (36). MR, fluorescence, and echocardiographic imaging demonstrated that these bifunctional nanoparticles improved localization of exogenous and endogenous therapeutic cells in infarcted rat myocardium and helped to preserve postinfarction cardiac structure and performance. The application of a local, external magnetic field to attract the superparamagnetic nanoparticles further enhanced particle localization and augmented cardiac functional improvements.

\section{PHARMACOKINETICS AND PARTICLE DESIGN CONSIDERATIONS}

The pharmacokinetics and biodistribution of nanoparticulate imaging media are ultimately critical to their diagnostic and therapeutic utility, as well as their safety. The creation of clinically useful tissue images relies on timely accumulation of sufficient particle quantities in target structures, as well as adequate blood clearance and minimal nonspecific uptake. Importantly, there are modality-specific considerations associated with these basic pharmacokinetic requirements for image formation. Although imaging with highly sensitive modalities reduces nanoparticle detection thresholds and improves target tissue signal strength, it also increases susceptibility to background noise from nonspecific uptake and inadequate blood pool clearance. Similarly, highly sensitive modalities are also more prone to false-positive detection from nanoparticles entrapped in off-target locations.

Although it is advantageous that the size, shape, and surface chemistry of nanoparticles can often be modified to adjust particle pharmacokinetics, it is important to consider that such alterations typically involve compromise. For example, nanoparticle surfaces are frequently modified with polyethylene glycol to decrease rates of nonspecific blood clearance and increase circulation times (24). Although this is generally favorable for targeted molecular binding and therapeutic delivery, the decreased rate of blood pool clearance tends to delay image acquisition and bioelimination. Furthermore, surface modification may also indirectly increase background signals by producing larger particles with lower levels of renal clearance and greater eventual MPS retention (26). For these reasons, the design of safe and clinically effective nanoparticulate imaging agents can be considerably challenging. Pharmacokinetic considerations are especially important in nuclear imaging because longer circulation times and slower tissue uptake rates can necessitate the use of radionuclides with long decay half-lives, which may increase radiation exposure. Examples of long-lived radionuclides used in preclinical imaging with nanoparticulate agents include ${ }^{64} \mathrm{Cu}$ (PET; half-life $\left.\left[\mathrm{t}_{1 / 2}\right]=12.7 \mathrm{~h}\right)(5,7,8,10,15,18),{ }^{89} \mathrm{Zr}$ $\left(\right.$ PET; $\left.\mathrm{t}_{1 / 2}=78.4 \mathrm{~h}\right)(6),{ }^{76} \mathrm{Br}\left(\mathrm{PET} ; \mathrm{t}_{1 / 2}=16.2 \mathrm{~h}\right)(9)$, and ${ }^{111}$ In (SPECT; $\left.\mathrm{t}_{1 / 2}=67.2 \mathrm{~h}\right)(2)$.

Ultimately, it is critical that diagnostic nanoparticles achieve timely and complete elimination or biodegradation after imaging and do so without producing toxic or immunogenic effects. From a safety standpoint, renal filtration is the preferred route for nanoparticle elimination because it is the quickest and most direct and thus minimizes chances for biologic interaction. However, rapid renal filtration $(<4 \mathrm{~h})$ tends to limit time for targeting to specific tissues and imposes strict constraints on nanoparticle design, as particles must have hydrodynamic diameters of less than $5.5 \mathrm{~nm}$ and zwitterionic or neutral surface charges (22). In contrast, larger nanoparticles are typically retained in MPS organs until they are degraded into smaller elements that can be further metabolized or excreted in bile or urine. This method of elimination is more variable and often increases exposure because it is considerably slower and often incomplete. Although rapid biliary clearance of larger nanoparticles $(\sim 250 \mathrm{~nm})$ occurs in rats, it is critical to note that this behavior has not been observed in large animals and humans (60). For these reasons, regulatory approval for nanoparticles has traditionally been restricted to organic or iron-based compositions that can be readily eliminated or safely metabolized (61).

Bioelimination is a significant challenge in the design of contrast media for molecular CT. Because of the relatively low sensitivity of CT, nanoparticles designed for this purpose typically contain large amounts of poorly metabolizable heavy metal crystals that are difficult to eliminate and potentially toxic. One possible solution to this is to use organic molecules to encapsulate large payloads of smallmolecule organometallic compounds (62). This allows for the creation of nanoparticles with considerable metal content that are easily broken down into smaller organic and organometallic compounds that can be excreted through the renal and biliary systems.

In all, given their low rates of extravascular tissue penetration and potential for extended circulation times, nanoparticles tend to be favorably suited for imaging entities within or near the vascular wall, such as blood, atheromas, thrombi, and angiogenic vessels (25). Nanoparticulate imaging agents also hold promise for applications such as stem cell tracking, ex vivo cell and tissue imaging, and imaging of MPS organs and pathologic tissues with permeable microvasculature. Importantly, the potential of nanoparticules in these applications is especially great when their imaging functionality is coupled to therapeutic delivery or radiologic intervention.

\section{CLINICAL TRANSLATION OF NANOPARTICLE TECHNOLOGY}

Despite the intriguing attributes of nanoparticles and significant research efforts to foster their development, 
nanoparticulate imaging agents have thus far achieved only limited clinical use $(25,61)$. This restricted advancement is due, in part, to the established effectiveness of smallmolecule formulations and the evolving scope of applications for which diagnostic and therapeutic nanoparticles are potentially suitable. However, most importantly, nanoparticulate agents have been saddled by pharmacokinetic limitations affecting background noise, acquisition times, reproducibility, and/or particle elimination (25). Despite more limited capabilities, small molecules generally have simpler compositions and more rapid elimination kinetics than nanoparticles and thus typically raise fewer safety, regulatory, and intellectual property concerns (61).

\section{CONCLUSION}

Nanoparticles exhibit size-based circulation, biodistribution, and elimination properties different from those of small molecules and microparticles and provide versatile platforms that can potentially be engineered to create both multimodal and multifunctional imaging agents with tunable properties. Despite their promise, nanoparticulate imaging agents have thus far attained very limited clinical use; fulfilment of their potential requires continued investment in the development of particle and instrumentation technology. Part 2 of this article, which will be published in a subsequent issue, provides a more detailed review of imaging with radiolabeled nanoparticles in preclinical models of cardiovascular disease.

\section{DISCLOSURE}

Dr. Stendahl is supported by NIH T32 training grant HL098069. No other potential conflict of interest relevant to this article was reported.

\section{REFERENCES}

1. Alwan A, Armstrong T, Bettcher D, et al. Global Status Report on Noncommunicable Diseases 2010. Geneva, Switzerland: World Health Organization; 2011.

2. Lukyanov AN, Hartner WC, Torchilin VP. Increased accumulation of PEG-PE micelles in the area of experimental myocardial infarction in rabbits. $J$ Controlled Release. 2004;94:187-193.

3. Urakami T, Kawaguchi AT, Akai S, et al. In vivo distribution of liposomeencapsulated hemoglobin determined by positron emission tomography. Artif Organs. 2009;33:164-168.

4. Hwang H, Kwon J, Oh P-S, et al. Peptide-loaded nanoparticles and radionuclide imaging for individualized treatment of myocardial ischemia. Radiology. 2014;273:160-167.

5. Luehmann HP, Pressly ED, Detering L, et al. PET/CT imaging of chemokine receptor CCR5 in vascular injury model using targeted nanoparticle. J Nucl Med. 2014;55:629-634.

6. Majmudar MD, Yoo J, Keliher EJ, et al. Polymeric nanoparticle PET/MR imaging allows macrophage detection in atherosclerotic plaques. Circ Res. 2013;112:755-761.

7. Liu Y, Pressly ED, Abendschein DR, et al. Targeting angiogenesis using a C-type atrial natriuretic factor-conjugated nanoprobe and PET. J Nucl Med. 2011;52: 1956-1963.

8. Fukukawa K-I, Rossin R, Hagooly A, et al. Synthesis and characterization of core-shell star copolymers for in vivo PET imaging applications. Biomacromolecules. 2008;9:1329-1339.

9. Almutairi A, Rossin R, Shokeen M, et al. Biodegradable dendritic positronemitting nanoprobes for the noninvasive imaging of angiogenesis. Proc Natl Acad Sci USA. 2009;106:685-690.
10. Seo JW, Baek H, Mahakian LM, et al. ${ }^{64} \mathrm{Cu}$-labeled LyP-1-dendrimer for PETCT imaging of atherosclerotic plaque. Bioconjug Chem. 2014;25:231-239.

11. Criscione JM, Dobrucki LW, Zhuang ZW, et al. Development and application of a multimodal contrast agent for SPECT/CT hybrid imaging. Bioconjug Chem. 2011;22:1784-1792.

12. Jung C, Kaul MG, Bruns OT, et al. Intraperitoneal injection improves the uptake of nanoparticle-labeled high-density lipoprotein to atherosclerotic plaques compared with intravenous injection: a multimodal imaging study in ApoE knockout mice. Circ Cardiovasc Imaging. 2014;7:303-311.

13. Morales-Avila E, Ferro-Flores G, Ocampo-Garcia BE, et al. Multimeric system of ${ }^{99 \mathrm{~m} T c-l a b e l e d ~ g o l d ~ n a n o p a r t i c l e s ~ c o n j u g a t e d ~ t o ~} \mathrm{c}[\operatorname{RGDfK}(\mathrm{C})]$ for molecular imaging of tumor $\alpha_{\nu} \beta_{3}$ expression. Bioconjug Chem. 2011;22:913-922.

14. Nahrendorf M, Keliher E, Marinelli B, et al. Detection of macrophages in aortic aneurysms by nanoparticle positron emission tomography-computed tomography. Arterioscler Thromb Vasc Biol. 2011;31:750-757.

15. Ueno T, Dutta P, Keliher E, et al. Nanoparticle PET-CT detects rejection and immunomodulation in cardiac allografts. Circ Cardiovasc Imaging. 2013;6: $568-573$.

16. Devaraj NK, Keliher EJ, Thurber GM, Nahrendorf M, Weissleder R. ${ }^{18} \mathrm{~F}$ labeled nanoparticles for in vivo PET-CT imaging. Bioconjug Chem. 2009;20: 397-401.

17. Lijowski M, Caruthers $\mathrm{S}, \mathrm{Hu} \mathrm{G}$, et al. High-resolution SPECT-CT/MR molecular imaging of angiogenesis in the Vx2 model. Invest Radiol. 2009;44: $15-22$.

18. Liu Z, Cai W, He L, et al. In vivo biodistribution and highly efficient tumour targeting of carbon nanotubes in mice. Nat Nanotechnol. 2007;2:47-52.

19. Lee J, Lee TS, Ryu J, et al. RGD peptide-conjugated multimodal $\mathrm{NaGdF}_{4}: \mathrm{Yb}^{3+}$ / $\mathrm{Er}^{3+}$ nanophosphors for upconversion luminescence, MR, and PET imaging of tumor angiogenesis. J Nucl Med. 2013;54:96-103.

20. Mitragotri S, Lahann J. Physical approaches to biomaterial design. Nat Mater. 2009;8:15-23.

21. Ernsting MJ, Murakami M, Roy A, Li SD. Factors controlling the pharmacokinetics, biodistribution and intratumoral penetration of nanoparticles. J Control Release. 2013;172:782-794.

22. Choi HS, Liu W, Misra P, et al. Renal clearance of quantum dots. Nat Biotechnol. 2007;25:1165-1170.

23. Liu D, Mori A, Huang L. Role of liposome size and RES blockade in controlling biodistribution and tumor uptake of $\mathrm{GM}_{1}$-containing liposomes. Biochim Biophys Acta. 1992;1104:95-101.

24. Hrkach J, Von Hoff D, Mukkaram Ali M, et al. Preclinical developmental and clinical translation of a PSMA-targeted docetaxel nanoparticle with a differentiated pharmacological profile. Sci Transl Med. 2012;4:128ra39.

25. Kiessling F, Mertens ME, Grimm J, Lammers T. Nanoparticles for imaging: top or flop? Radiology. 2014;273:10-28.

26. Choi HS, Liu W, Liu F, et al. Design considerations for tumour-targeted nanoparticles. Nat Nanotechnol. 2010;5:42-47.

27. Rizvi SB, Ghaderi S, Keshtgar M, Seifalian AM. Semiconductor quantum dots as fluorescent probes for in vitro and in vivo bio-molecular and cellular imaging. Nano Rev. 2010;1:5161.

28. Stirrat CG, Newby DE, Robson JMJ, Jansen MA. The use of superparamagnetic iron oxide nanoparticles to assess cardiac inflammation. Curr Cardiovasc Imaging Rep. 2014;7:9263.

29. Jaque D, Martinez Maestro L, del Rosal B, et al. Nanoparticles for photothermal therapies. Nanoscale. 2014;6:9494-9530.

30. Cormode DP, Skajaa T, van Schooneveld MM, et al. Nanocrystal core high-density lipoproteins: a multimodality contrast agent platform. Nano Lett. 2008;8: 3715-3723.

31. Chen IY, Wu JC. Cardiovascular molecular imaging: focus on clinical translation. Circulation. 2011;123:425-443.

32. Winter P, Athey P, Kiefer G, et al. Improved paramagnetic chelate for molecular imaging with MRI. J Magn Magn Mater. 2005;293:540-545.

33. Ragheb RR, Kim D, Bandyopadhyay A, et al. Induced clustered nanoconfinement of superparamagnetic iron oxide in biodegradable nanoparticles enhances transverse relaxivity for targeted theranostics. Magn Reson Med. 2013;70: $1748-1760$.

34. Schmieder AH, Winter PM, Williams TA, et al. Molecular MR imaging of neovascular progression in the $\mathrm{Vx} 2$ tumor with $\alpha_{\nu} \beta_{3}$-targeted paramagnetic nanoparticles. Radiology. 2013;268:470-480.

35. Chen W, Cormode DP, Vengrenyuk Y, et al. Collagen-specific peptide conjugated HDL nanoparticles as MRI contrast agent to evaluate compositional changes in atherosclerotic plaque regression. JACC Cardiovasc Imaging. 2013;6: 373-384.

36. Cheng K, Shen D, Hensley MT, et al. Magnetic antibody-linked nanomatchmakers for therapeutic cell targeting. Nat Commun. 2014;5:4880. 
37. Hyafil F, Cornily JC, Feig JE, et al. Noninvasive detection of macrophages using a nanoparticulate contrast agent for computed tomography. Nat Med. 2007;13: 636-641.

38. Winter PM, Shukla HP, Caruthers SD, et al. Molecular imaging of human thrombus with computed tomography. Acad Radiol. 2005;12(suppl): S9-S13.

39. Danila D, Johnson E, Kee P. CT imaging of myocardial scars with collagentargeting gold nanoparticles. Nanomedicine. 2013;9:1067-1076.

40. Anderson NG, Butler AP. Clinical applications of spectral molecular imaging: potential and challenges. Contrast Media Mol Imaging. 2014;9:3-12.

41. Cormode DP, Roessl E, Thran A, et al. Atherosclerotic plaque composition: analysis with multicolor CT and targeted gold nanoparticles. Radiology. 2010; 256:774-782.

42. Zainon R, Ronaldson JP, Janmale T, et al. Spectral CT of carotid atherosclerotic plaque: comparison with histology. Eur Radiol. 2012;22:2581-2588.

43. Bhavane R, Badea C, Ghaghada KB, et al. Dual-energy computed tomography imaging of atherosclerotic plaques in a mouse model using a liposomal-iodine nanoparticle contrast agent. Circ Cardiovasc Imaging. 2013;6:285-294.

44. Pan D, Schirra CO, Wickline SA, Lanza GM. Multicolor computed tomographic molecular imaging with noncrystalline high-metal-density nanobeacons. Contrast Media Mol Imaging. 2014;9:13-25.

45. Chen F, Ehlerding EB, Cai W. Theranostic nanoparticles. J Nucl Med. 2014;55: 1919-1922.

46. Winter PM, Neubauer AM, Caruthers SD, et al. Endothelial $\alpha_{\nu} \beta_{3}$ integrin-targeted fumagillin nanoparticles inhibit angiogenesis in atherosclerosis. Arterioscler Thromb Vasc Biol. 2006;26:2103-2109.

47. Lobatto ME, Fayad ZA, Slivera S, et al. Multimodal clinical imaging to longitudinally assess a nanomedical anti-inflammatory treatment in experimental atherosclerosis. Mol Pharm. 2010;7:2020-2029.

48. Duivenvoorden R, Tang J, Cormode DP, et al. A statin-loaded reconstituted highdensity lipoprotein nanoparticle inhibits atherosclerotic plaque inflammation. Nat Commun. 2014;5:3065.

49. Winter PM, Caruthers SD, Allen JS, et al. Molecular imaging of angiogenic therapy in peripheral vascular disease with $\alpha_{\nu} \beta_{3}$-integrin-targeted nanoparticles. Magn Reson Med. 2010;64:369-376.

50. Fujii $\mathrm{H}, \mathrm{Li} \mathrm{SH}, \mathrm{Wu} \mathrm{J}$, et al. Repeated and targeted transfer of angiogenic plasmids into the infarcted rat heart via ultrasound targeted microbubble destruction enhances cardiac repair. Eur Heart J. 2011;32:2075-2084.
51. Harel-Adar T, Ben Mordechai T, Amsalem Y, Feinberg MS, Leor J, Cohen S. Modulation of cardiac macrophages by phosphatidylserine-presenting liposomes improves infarct repair. Proc Natl Acad Sci USA. 2011;108:1827-1832.

52. Leor J, Rozen L, Zuloff-Shani A, et al. Ex vivo activated human macrophages improve healing, remodeling, and function of the infarcted heart. Circulation. 2006;114:I94-I100.

53. Fredman G, Kamaly N, Spolitu S, et al. Targeted nanoparticles containing the proresolving peptide Ac2-26 protect against advanced atherosclerosis in hypercholesteremic mice. Sci Transl Med. 2015;7:275ra20.

54. Katsuki S, Matoba T, Nakashiro S, et al. Nanoparticle-mediated delivery of pitavastatin inhibits atherosclerotic plaque destabilization/rupture in mice by regulating the recruitment of inflammatory monocytes. Circulation. 2014;129: 896-906.

55. Nagahama R, Matoba T, Nakano K, Kim-Mitsuyama S, Sunagawa K, Egashira K. Nanoparticle-mediated delivery of pioglitazone enhances therapeutic neovascularization in a murine model of hindlimb ischemia. Arterioscler Thromb Vasc Biol. 2012;32:2427-2434.

56. Leuschner F, Dutta P, Gorbatov R, et al. Therapeutic siRNA silencing in inflammatory monocytes in mice. Nat Biotechnol. 2011;29:1005-1010.

57. Bartlett DW, Su H, Hildebrandt IJ, Weber WA, Davis ME. Impact of tumorspecific targeting on the biodistribution and efficacy of siRNA nanoparticles measured by multimodality in vivo imaging. Proc Natl Acad Sci USA. 2007; 104:15549-15554.

58. Kirpotin DB, Drummond DC, Shao Y, et al. Antibody targeting of longcirculating lipidic nanoparticles does not increase tumor localization but does increase internalization in animal models. Cancer Res. 2006;66:6732-6740.

59. Andresen TL, Jensen SS, Jorgensen K. Advanced strategies in liposomal cancer therapy: problems and prospects of active and tumor specific drug release. Prog Lipid Res. 2005;44:68-97.

60. Bulte JW, Schmieder AH, Keupp J, Caruthers SD, Wickline SA, Lanza GM. MR cholangiography demonstrates unsuspected rapid biliary clearance of nanoparticles in rodents: implications for clinical translation. Nanomedicine. 2014;10: 1385-1388.

61. Choi HS, Frangioni JV. Nanoparticles for biomedical imaging: fundamentals of clinical translation. Mol Imaging. 2010;9:291-310.

62. Pan D, Williams TA, Senpan A, et al. Detecting vascular biosignatures with a colloidal, radio-opaque polymeric nanoparticle. J Am Chem Soc. 2009;131: $15522-15527$. 